\title{
Le texte cruel, «Cahiers de littérature française» III, a cura di Franca Franchi
}

\section{Luigi Luison}

\section{(2) OpenEdition}

1 Journals

\section{Edizione digitale}

URL: https://journals.openedition.org/studifrancesi/27003

DOI: 10.4000/studifrancesi.27003

ISSN: 2421-5856

\section{Editore}

Rosenberg \& Sellier

\section{Edizione cartacea}

Data di pubblicazione: 1 avril 2007

Paginazione: 230-231

ISSN: 0039-2944

\section{Notizia bibliografica digitale}

Luigi Luison, «Le texte cruel, «Cahiers de littérature française» III, a cura di Franca Franchi», Studi

Francesi [Online], 151 (LI | I) | 2007, online dal 30 novembre 2015, consultato il 23 novembre 2021

URL: http://journals.openedition.org/studifrancesi/27003 ; DOI: https://doi.org/10.4000/studifrancesi. 27003

Questo documento è stato generato automaticamente il 23 novembre 2021.

\section{(c)}

Studi Francesi è distribuita con Licenza Creative Commons Attribuzione - Non commerciale - Non opere derivate 4.0 Internazionale. 


\title{
Le texte cruel, «Cahiers de littérature française» III, a cura di Franca Franchi
}

\author{
Luigi Luison
}

\section{NOTIZIA}

Aa. Vv., Le texte cruel, «Cahiers de littérature française» III, a cura di Franca FRANCHI, Bergamo University Press, Edizioni Sestante, e Paris, L'Harmattan, juin 2006, pp. 131.

1 I saggi raccolti in questo volume, frutto di un convegno sul "testo crudele", si interrogano sulle ragioni e sulle tipologie della crudeltà. L'obiettivo degli Autori è quello di abbozzare una geografia delle metamorfosi di una cultura della crudeltà, che dopo Baudelaire, ha passato il testimone alla nostra modernità.

Dalla definizione degli interni borghesi che si modellano con la personalità di coloro che li abitano, e dalla teoria di Jules Janin sulla relazione tra la ristrettezza degli spazi del mondo borghese e la vocazione al crimine, si sviluppa l'articolo di Alberto CASTOLDI (L'Espace cruel, pp. 7-19) che delinea uno spazio chiuso inteso come metafora dello spazio interiore in cui albergano le pulsioni più indicibili. Il carattere archetipico di questa immagine è presente ne $L a$ Fanfarlo di Baudelaire. L'A. mette in evidenza l'anatomia della camera, alla quale appartiene un corpo, come l'anatomia stessa della poesia che lo genera. In questo modo la poesia diventa un corpo che soffre e il poeta è il cerimoniere di questo rito funebre. Il percorso tracciato dall'A. diventa un percorso della modernità centrato sul corpo sofferente, collocato in una sorta di sala anatomica. Alberto Castoldi si sposta poi su La Chevelure di Maupassant, in cui lo spazio crudele prende l'aspetto di un mobile antico che racchiude un corpo femminile, come una specie di "custodia" che rimanda all'abitazione. Parallelamente, però, l'A. individua un altro percorso che disegna una prospettiva capovolta, dove il femminile è lo "spazio crudele" del maschile, spazio in cui il maschio proietta le proprie pulsioni. Ecco che 
allora La tana angosciante di Kafka non sarà più uno spazio crudele qualsiasi all'interno del mondo borghese, ma diventa un'atroce metafora esistenziale. Secondo Castoldi, dobbiamo prendere coscienza che la tana siamo noi e che siamo prigionieri di noi stessi; è il caso di Le Horla di Maupassant: "noi siamo lo spazio crudele della nostra sofferenza".

Il secolo dei Lumi e il tema del "cœur mangé" è l'argomento trattato da Michel DELON (Cœurs mangés. Cruauté et ironie au tournant des Lumières, pp. 21-30) che ripercorre sommariamente i lavori di Mariella Di Maio sull'argomento. Dalla tradizione medievale e rinascimentale, che ha generato in tutta Europa i racconti in cui la passione amorosa clandestina genera banchetti antropofagi, l'A. si sofferma maggiormente sull'epoca settecentesca, dove Gabrielle de Vergy di De Belloy e Fayel di Baculard D'Arnaud illustrano molto bene un processo che, rispetto ai secoli precedenti, rivela una diminuzione dell'orrore e una interiorizzazione della crudeltà, conforme al gusto dei tempi. Bisognerà attendere De Sade per assistere ad un ritorno crudele dell'antropofagia. Michel Delon sottolinea che attraverso tutte le mutazioni subite dal rito cannibale, la doppia immagine del cuore strappato dal petto e il suo divoramento, mantiene una violenza legata alle ossessioni più profonde dell'uomo: coloro che riprendono la vita si sostituiscono a coloro che la danno e l'amore si ritorce in cannibalismo.

4 La curatrice del volume, Franca FRANCHI disegna, invece, un percorso che parte dall'Ottocento ed arriva sino ai nostri giorni e che ha per tema il corpo come opera d'arte (Dans la chair et sur la peau: l'écriture de la modernité, pp. 31-45). De la mode di Théophile Gautier e Le Peintre de la vie moderne di Baudelaire, sono esempi di una letteratura che attesta il modo in cui il corpo "alla moda" diventa l'oggetto privilegiato di una riflessione sullo statuto dell'opera d'arte e sul dialogo tra arte e modernità. $\mathrm{E}$ partendo proprio dall'idea che il corpo si fa vestito, il lavoro che si opera su di esso diventa l'oggetto di una serie di esperienze crudeli: diventando opera d'arte, il corpo esibisce la sofferenza, l'oltraggio e la profanazione. L'opera dell'artista Hans Bellmer delinea chiaramente questo procedimento: con lui, il corpo si presenta come luogo tragico del desiderio e raggiunge il limite estremo delle sue possibilità nella violenza delle sue trasformazioni. La Body Art è la versione moderna dei manichini smantellati di Bellmer o delle fotografie rielaborate di Molinier, che traduce in immagine dei corpifeticci in movimento; oppure la riflessione artistica di Orlan che reinterpreta l'arte di Marcel Duchamp, in cui è la pelle "a strati" ad essere l'elemento dal quale si sviluppa la sua creatività: il primo strato è formato dall'armatura metallica che riproduce le curve femminili; il secondo strato è dato dal cuoio applicato sull'armatura; mentre la pittura di colore rosa rappresenta il terzo strato di pelle che simula l'epidermide.

Dalla pittura che vuole imitare la pelle del corpo, passiamo alla pittura surrealista con il primo dei tre lavori presentati da Marina GALLETTI (Georges Bataille ou la littérature comme «exercice de cruauté», pp. 47-61), incentrato sulla teoria di Georges Bataille, secondo cui la pittura contemporanea prolunga la preoccupazione di ogni religione, consistente nell'aprire gli uomini all'idea di una coscienza della morte, attraverso la pratica sanguinosa del sacrificio. Questa teoria è espressa molto bene in Les Larmes d'Éros, un vero teatro di crudeltà, dove l'immagine di un antico sacrificio atzeco fissato nella miniatura di un manoscritto è unita all'immagine moderna di due raccolte fotografiche, il cui montaggio ha per effetto quello di introdurci all'esperienza del sacro e alla sua espulsione dalla sfera cristiana. Georges Bataille approfondisce tale concetto con Hegel, 
la mort et le sacrifice, in cui analizza il rapporto tra la coscienza della morte e la necessità di ricorrere alla rappresentazione come unica possibilità per l'uomo di accedere alla coscienza di sé.

6 Il secondo articolo presentato da Marina Galletti riguarda una conferenza, inedita sino ad oggi, di Georges BATAILLE sulla figura quattrocentesca di Gilles de Rais (Gilles de Rais, pp. 63-80). Bataille introduce la storia del "più spregevole criminale di tutti i tempi", paragonandolo a criminali famosi del xx secolo, in particolare Friedrich Haarmann, un criminale sospettato di cannibalismo e ghigliottinato nel 1925, e Peter Kurten, emule dei personaggi di De Sade e ghigliottinato nel 1932. Bataille collega la storia di Gilles de Rais alle idee sulla cavalleria riportate nei trattati dell'epoca, imbevuti dell'insegnamento cristiano. Grande esperto di storia e letteratura medievale, Bataille riconduce le origini della cavalleria a società segrete germaniche.

7 Il terzo e ultimo contributo di Marina Galletti si riferisce ad un altro documento prezioso (Rapport de M. Georges Bataille, élève de l'École des chartes, au sujet de ses travaux pendant son séjour à la Maison de l'Institut de France à Londres, pp. 81-84) redatto da Bataille in gioventù, prima della redazione dei sui primi scritti, La Châtelaine Gentiane e Ralph Webb. Questo documento è il resoconto di una ricerca effettuata da Bataille durante il soggiorno londinese su una chanson de geste scoperta agli inizi del Novecento, La Chanson de Guillaume. L'esperienza londinese, come rivela Marina Galletti, sarebbe stata cruciale per Bataille, poiché avrebbe provocato nello scrittore la perdita della fede religiosa, come lui stesso confesserà più tardi nella "Notice autobiographique".

Baudelaire visto da Proust è l'argomento dell'articolo di André GUYAU (Baudelaire, «cruel avec infiniment de sensibilité», pp. 85-91). Proust, nel tentativo di convincere la madre ad amare la poesia di Baudelaire, solleva la questione della crudeltà e, come si evince da Contre Sainte-Beuve, sostiene che il poeta è sensibile alla sofferenza e la sua sensibilità è il suo modo di essere "crudele". Leggere Les Fleurs du mal significa interagire con due letture, quella che permette di accedere alla sensibilità dell'uomo e quella che concepisce l'insensibilità dell'artista.

9 Liana NISSIM (L'écriture de la cruauté dans le roman africain francophone, pp. 93-109) ci offre una panoramica sul tema della violenza nella letteratura africana. L'A. introduce l'argomento citando gli atti di un convegno del 1990 pubblicati a cura di Franca Marcato sul tema Figures et fantasmes de la violence dans les littératures francophones de l'Afrique subsaharienne da cui risulta non solo che il tema della violenza è centrale nelle letterature dell'Africa francofona, ma anche che la scrittura vi si fa volontariamente crudele, allo scopo di scuotere il mondo intero sulla condizione spaventosa in cui si trova il continente. La violenza del linguaggio di alcuni scrittori contemporanei è il risultato di una scelta precisa operata dal romanzo africano che, rispetto agli anni ' 20 , ha subìto una grande trasformazione, conquistando una posizione autonoma rispetto al modello francese e coinvolgendo tutti gli aspetti strutturali propri della scrittura romanzesca. Gli artefici di questa trasformazione sono due romanzi del 1968, Les Soleils des indépendances di Ivoirien Ahmadou e Le Devoir de violence di Malien Yambo Ouologuem. In questi testi la crudeltà appare non solo nello sviluppo delle tematiche, ma soprattutto nella violenza esercitata sulla scrittura. Da quella data, il romanzo africano non ha fatto altro che continuare a cercare forme nuove per raccontare l'orrore del continente; ne sono un esempio L'Aîné des orphelins di Guinéen Tierno Monénembo imperniato sul genocidio del Rwanda e Allah n'est pas obligé di Ahmadou Kourouma che racconta gli orrori delle guerre civili della Sierra Leone e della Liberia. 
10 "La crudeltà del testo comincia dalla copertina del libro", afferma nel suo articolo Jean DE PALACIO (Un brelan de tortionnaires. Émile Goudeau, Laurent Tailhade, Léon Bloy, pp. 111-122), il quale prende in esame la crudeltà retorica ed aggressiva che traspare da tre racconti: Fleurs du bitume di Émile Goudeau, Imbéciles et gredins di Laurent Tailhade e Propos d'un entrepreneur de démolitions di Léon Bloy.

11 L'articolo che chiude questo volume sul testo crudele riguarda la figura di d'Albert, il protagonista di Mademoiselle de Maupin di Théophile Gautier, ed è a firma di Paolo TORTONESE (Théophile Gautier et le déclin de la sympathie esthétique, pp. 123-131). Ciò che l'A. intende evidenziare, concerne gli spettacoli crudeli che il protagonista mette in scena nel suo teatro interiore, in opposizione alla sua piatta esistenza. D'Albert si contempla nello specchio di un'antichità che il cristianesimo non aveva ancora contagiato con l'ipocrisia spirituale e con la condanna della carne. "Ma in questo specchio, accanto ad un edonismo raggiante, trova le ombre della depravazione". Il messaggio di Gautier, secondo l'A., è chiaro: occorre che ognuno di noi trovi in un'immagine il suo desiderio più segreto, per comprenderlo. I personaggi di Gautier non cessano mai di invocare una bellezza ideale, ispirata dall'arte, ma che è strettamente soggettiva, individuale e, forse, un sogno egoista. 